\title{
SOME AMALGAM STRUCTURES FOR BIANCHI GROUPS
}

\author{
CHARLES FROHMAN AND BENJAMIN FINE
}

(Communicated by Bhama Srinivasan)

\begin{abstract}
Splittings as an amalgamated sum, where one of the factors is the projective elementary matrices, are displayed for all the Bianchi groups except for the Euclidian ones. Also splittings as an HNN group, where the base is $\mathrm{PSL}_{2}(Z)$, are given for all the Bianchi groups except $\mathrm{PSL}_{2}\left(O_{1}\right)$ and $\mathrm{PSL}_{2}\left(\mathrm{O}_{3}\right)$.
\end{abstract}

0. Introduction. This paper grew out of a seminar on the Bianchi groups. In addition to the authors, Seymour Bachmuth and Morris Newman were active participants. Without their knowledge and enthusiasm this paper would not exist.

Our knowledge of the modular group stands as the basic example upon which the study of infinite groups is modeled. Since $\mathrm{PSL}_{2}(\mathbf{Z})$ is a discrete subgroup of $\mathrm{PSL}_{2}(\mathbf{R})$ we can view the modular group as a group of isometries of the hyperbolic plane $H^{2}$ acting in a properly discontinuous fashion. The interplay between the number theory of the rational integers and the geometry of $\mathrm{PSL}_{2}(\mathbf{Z}) \backslash H^{2}$ has led to the beautiful theory of the modular group. Let $d$ be a positive square free integer, and let $O_{d}$ denote the ring of integers in the field $\mathbf{Q}(\sqrt{-d})$. After the rational integers, the quadratic imaginary number rings $O_{d}$ are the most completely understood examples in number theory. The groups $\Gamma_{d}=\mathrm{PSL}_{2}\left(O_{d}\right)$ are known as the Bianchi groups and have been extensively studied. Since $\Gamma_{d}$ is a discrete subgroup of $\mathrm{PSL}_{2}(\mathbf{C})$ we can view the Bianchi groups as isometries of hyperbolic space $H^{3}$ acting in a properly discontinuous fashion. Hence one would expect that the geometry of $\Gamma_{d} \backslash H^{3}$ and the number theory of $O_{d}$ could be used to derive information about $\Gamma_{d}$ in much the same way that the modular group has been studied. In fact study of the geometry of $\Gamma_{d}$ goes back to Bianchi [B1], although the work of Swan [Sw] is the fundamental reference for modern studies. In [R], Riley describes a computer program based on Swan's work that computes the Ford domains of the Bianchi groups. Also Hatcher $[\mathbf{H}]$ has drawn the orbifolds of $\mathrm{PGL}_{2}\left(O_{d}\right)$ for all $d<100$.

The question that has driven research in this direction in recent years is the cuspidal cohomology question. Simply stated the question is: For which $d$ is the first rational homology of $\Gamma_{d} \backslash H^{3}$ carried by the cusps? This question has been answered in the papers [B, GS, Ro, V and Z]. Specifically the answer is when $d \in\{1,2,3,5,6,7,11,15,19,23,31,39,47,71\}$.

In this paper we use results appearing in $[\mathbf{S w}]$ to show the existence of a family of nontrivial splittings of $\Gamma_{d}$. Throughout this paper $\{1, \omega\}$ will denote the standard module basis for $O_{d}$. The projective elementary group $E_{d}$ is the subgroup of $\Gamma_{d}$ generated by $a=-1 / z, t=z+1$ and $\mu=z+\omega$. If $O_{d}$ is not Euclidean then we

Received by the editors June 24, 1986 and, in revised form, October 15, 1986.

1980 Mathematics Subject Classification (1985 Revision). Primary 20E06, 57M99. 
show that $\Gamma_{d} \cong E_{d} *_{F_{d}} G_{d}$ where $F_{d}$ is a subgroup of $E_{d}$ that is canonically defined and $G_{d}$ is a group whose structure depends on $d$. This splitting comes from an incompressible surface in $\Gamma_{d} \backslash H^{3}$, which in contrast to the splittings in $[\mathbf{H}, \mathbf{Z}]$ is not totally geodesic. The basic fact that leads to the preceding result is that for $d \neq 1$ or $3, \Gamma_{d}$ is an $\mathrm{HNN}$ group amalgamated over $\mathrm{PSL}_{2}(\mathbf{Z})$. We also find splittings of $\Gamma_{2}$, $\Gamma_{7}$, and $\Gamma_{11}$ as amalgamated sums. It is known that $\Gamma_{1}$ splits as an amalgamated sum over $\mathrm{PSL}_{2}(\mathbf{Z})$ and that $\Gamma_{3}$ does not admit splittings as an amalgamated sum [S].

The following theorem appears in [CS].

THEOREM. Let $M$ be a compact, connected, orientable 3-manifold having a nonempty family of tori as boundary. Suppose that $H_{1}(\partial M, \mathbf{Q}) \rightarrow H_{1}(M, \mathbf{Q})$ is surjective and that $M$ is not homeomorphic to $D^{2} \times S^{1}$ or $S^{1} \times S^{1} \times I$. Then $M$ contains a separating, (properly embedded) incompressible surface which has nonempty boundary and is not boundary parallel.

The existence of a separating incompressible surface that is not boundary parallel implies that $\pi_{1}(M)$ splits as an amalgamated sum. If $\Gamma$ is a discrete subgroup of $\mathrm{PSL}_{2}(\mathbf{C})$ and $\Gamma \backslash H^{3}$ has finite volume, then $\Gamma \backslash H^{3}$ is homeomorphic to the interior of a compact three-manifold. The rational homology of $\Gamma \backslash H^{3}$ is naturally isomorphic to the rational homology of $\Gamma$. Hence it makes sense to refer to the peripheral homology of $\Gamma$ (homology coming from the boundary). From the solution of the cuspidal cohomology problem we know that only 14 Bianchi groups have the property that their rational first homology is generated by their peripheral homology. Hence only for $d \in\{2,5,6,7,11,15,19,23,31,39,47,71\}$ does Culler and Shalen's proof adapt to show that $\Gamma_{d}$ is an amalgamated sum. It was out of a desire to sharpen their results that we took about to understand the other Bianchi groups.

1. Basic concepts. Realize $H^{3}$ as $\{(z, \varsigma) \mid z, \varsigma \in \mathbf{C}, \varsigma>0\}$ using the standard upperhalf space metric. The group of orientation preserving isometries of $H^{3}$ is isomorphic to $\mathrm{PSL}_{2}(\mathbf{C})$ where the action is given as follows. Suppose $\sigma=\left(\begin{array}{ll}a & b \\ c & d\end{array}\right) \in$ $\mathrm{SL}_{2}(\mathbf{C})$ represents an element of $\mathrm{PSL}_{2}(\mathbf{C})$. Then $\sigma(z, \varsigma)=\left(z^{\prime}, \varsigma^{\prime}\right)$, where

$$
z^{\prime} \frac{(\bar{d}-\bar{c} \bar{z})(a z-b)-\varsigma^{2} \bar{c} a}{|c z-d|^{2}+\varsigma^{2}|c|^{2}} \quad \text { and } \quad \varsigma^{\prime}=\frac{\varsigma}{|c z-d|^{2}+\varsigma^{2}|c|^{2}} \text {. }
$$

If $\Gamma$ is a discrete subgroup of $\mathrm{PSL}_{2}(\mathbf{C})$ then the Ford domain $B$ of $\Gamma$ is the region of $H^{3}$ where all $\sigma \in \Gamma$ have Jacobian determinant less than or equal to one. If $\Phi$ is the subgroup of $\Gamma$ that fixes infinity and if $D$ is a fundamental domain for $\Phi$ then $B \cap D$ is a fundamental domain for $\Gamma$. In [Sw], a method is given for computing the Ford domains of the Bianchi groups. Specifically

$$
B=\left\{\left(z, \varsigma|| \mu z-\left.\lambda\right|^{2}+\varsigma^{2}|\mu|^{2} \geq 1 \forall \lambda, \mu \in O_{d},(\lambda, \mu)=O_{d}\right\},\right.
$$

where $(\lambda, \mu)=O_{d}$ means that $\lambda$ and $\mu$ generate $O_{d}$. Furthermore [Sw] it is shown that over the line $\operatorname{im} z=0$ the lower boundary of $B$ is made up of the spheres of radius one centered at the integers. More precisely $B \cap\{\operatorname{im} z=0\}=\{(x, \varsigma) \in$ $\left.\mathbf{R} \times \mathbf{R}_{>0}: \forall n \in \mathbf{Z},|x-n|^{2}+\varsigma^{2} \geq 1\right\}$.

If $\Gamma$ acts properly discontinuously on $H^{2}$ or $H^{3}$ then $\Gamma \backslash H^{n}$ inherits the structure of an orbifold [Th]. Although the orbifold concept clarifies the nature of our studies, the geometric and topological preliminaries at this state of development are rather 


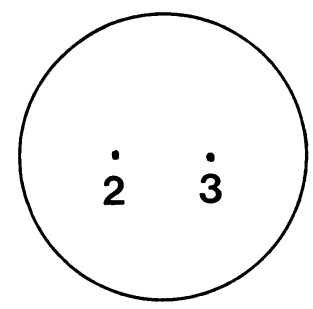

FIGURE 1

forboding. Hence we choose to follow Zieschang $[\mathbf{Z 1}]$ and state our results inside the framework of factor manifolds.

DEFINITION 1.1. Let $M$ be a manifold, with or without boundary, of dimension $n=2$ or 3 . Let $S$ be a codimension $2 \mathrm{CW}$-complex that is properly embedded in $M$. Assume also that each $n-2$ cell of $S$ has a positive integer assigned to it. We call $S$ the branch set of $M$. Call the number $h_{i}$ assigned to the cell $e_{i}$ of $S$ the index of $e_{i}$. We call the pair $(M, S)$ a factor manifold.

EXAMPLE 1.2. If $\Gamma$ is a Fuchsian group then $F=\Gamma \backslash H^{2}$ is a surface. The points on $F$ that correspond to points in $H^{2}$ having nontrivial stabilizers will make up the branch set $T$. To each $q \in T$ assign the order of the stabilizer of a lift of $q$ to $H^{2}$. In Figure 1 we have pictured the factor manifold corresponding to $\mathrm{PSL}_{2}(\mathbf{Z}) \backslash H^{2}$.

EXAMPLE 1.3. If $\Gamma$ is a discrete subgroup of $\mathrm{PSL}_{2}(\mathbf{C})$ then $M=\Gamma \backslash H^{3}$ is a three-manifold. We let the branch set $S$ be the subset of $M$ corresponding to points in $H^{3}$ having nontrivial stabilizers. We can give $S$ a cell structure by letting the vertices be the nonmanifold points of $S$ and the 1-cells be the arcs running between vertices. If some component of $S$ is a circle then just choose an arbitrary point on that component to be a vertex. Once again the index of a 1-cell $e$ is the order of the stabilizer of one of its lifts to $H^{3}$. In Figure 2 we have pictured the factor manifolds corresponding to $\Gamma_{1}, \Gamma_{2}, \Gamma_{3}, \Gamma_{5}, \Gamma_{6}, \Gamma_{7}, \Gamma_{11}$.

Suppose that $\Gamma$ is a discrete group of orientation preserving isometries of $H^{2}$ or $H^{3}$. We would like to use the Seifert-Van Kampen theorem in our study of $\Gamma$; hence we construct a complex that has $\Gamma$ as its fundamental group. At this point in our discussion we need to deal with surfaces and three-manifolds separately. First we will deal with factor surfaces, then with factor three-manifolds.

DEFINITION 1.4. Let $(F, T)$ be a factor surface. Replace a regular neighborhood $N\left(e_{i}\right)$ of each 0 -cell $e_{i}$ in $T$ by a disk $D_{i}$ so that the gluing map $H_{i}: \partial D_{i} \rightarrow \partial N\left(e_{i}\right)$ has degree $h_{i}$, the index of $e_{i}$. Denote the complex we have constructed by $\tilde{F}$.

In $[\mathrm{Z1}]$ it is remarked that if $(F, T)$ is the factor manifold corresponding to a discrete group of isometries $\Gamma$ of $H^{2}$ then $\pi_{1}(\tilde{F}) \cong \Gamma$.

DEFINITION 1.5. Let $(M, S)$ be a factor three-manifold. Let $N(S)$ be a regular neighborhood of $S$ in $M$. We can decompose $N(S)$ into balls and beams. The balls are regular neighborhoods of the 0-cells of $S$ in $M$. The beams are regular neighborhoods in the complement of the balls of the 1-cells of $S$ (see Figure 3). This decomposition of $N(S)$ induces a decomposition of $\partial N(S)$ into annuli that are the frontiers of beams in $\hat{M}=\mathrm{Cl}(M-N(S))$ and planar surfaces which are the frontiers of balls in $\hat{M}$. Denote by $A_{i}$ the annulus corresponding to the 1-cell $e_{i}$. 

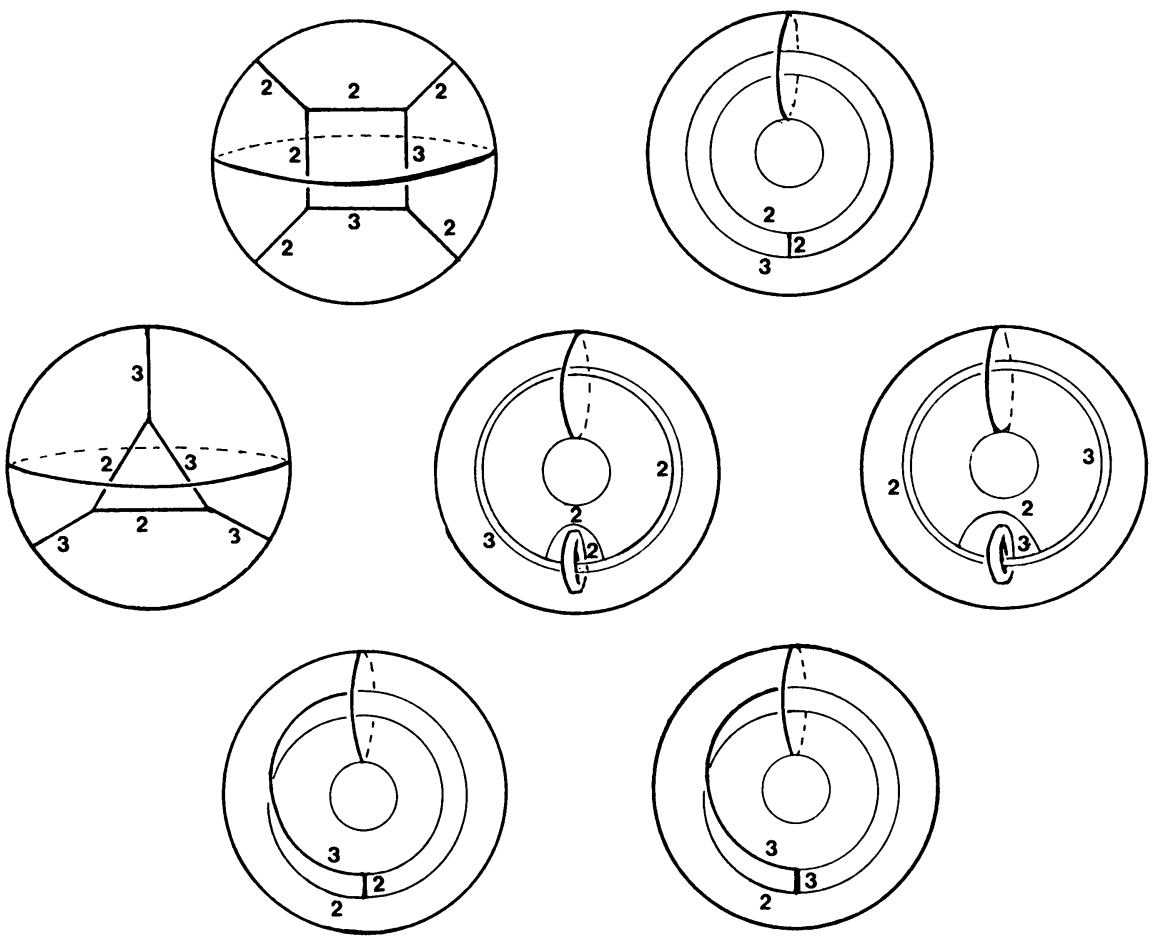

FigURE 2. From left to right, top to bottom, the factor manifolds corresponding to $\Gamma_{1}, \Gamma_{2}, \Gamma_{3}, \Gamma_{5}, \Gamma_{6}, \Gamma_{7}, \Gamma_{11}$.

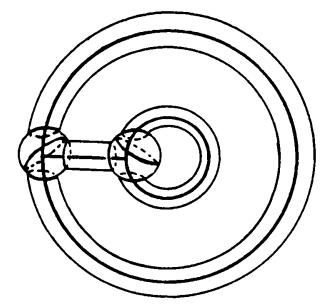

FIGURE 3

Let $H_{i}: \partial D^{2} \times I \rightarrow A_{i}$ be a map of degree $h_{i}$. Let $\tilde{M}$ be the complex obtained by glueing a copy of $D^{2} \times I$ onto $\hat{M}$ for each $A_{i}$ using the maps $H_{i}$ as glueing maps.

PROPOSITION 1.6. Let $\Gamma$ be a discrete subgroup of isometries of $H^{3}$. Let $(M, S)$ be the factor manifold corresponding to $\Gamma$. Then $\Gamma \cong \pi_{1}(\tilde{M})$.

ProOF. Let $q: H^{3} \rightarrow M$ be the quotient map. The restriction of $q$ to the complement $\hat{H}$ of $q^{-1}(\stackrel{\circ}{N}(S))$ is a regular covering of $\hat{M}$. Hence $\Gamma \approx \pi_{1}(\hat{M}) / q_{\#} \pi_{1}(\hat{H})$. Let $x_{i}$ be a meridian of the 1-cell $e_{i}$. To construct such a loop, run an arc $a_{i}$ that misses $N(S)$ from the basepoint out to the boundary of a small disk $D_{i}$ that intersects $S$ transversely in a single point lying in the interior of $e_{i}$, around the boundary of $D_{i}$ and back along $a_{i}$. The image of $\pi_{1}(\hat{H})$ in $\pi_{1}(\hat{M})$ is normally 
generated by $x_{i}^{h_{i}}$ where $i$ ranges over the indices of 1-cells of $S$. By construction $\pi_{1}(M)$ is isomorphic to $\Gamma$.

DEFINITION 1.7. Let $F$ be a surface that is embedded in the factor threemanifold $(M, S)$. If $F$ intersects $S$ transversely and misses the 0 -cells of $S$, then we say that $F$ is embedded nicely with respect to $S$. We obtain the induced factor manifold structure on $F$ by letting the branch set $T$ be the set of points $F \cap S$ equipped with the ramification indices of the corresponding 1-cells of $S$. Notice that if care is taken in the construction of $\tilde{F}$ and $\tilde{M}$ then $\tilde{F}$ can be viewed as a subcomplex of $\tilde{M}$. We say that $F$ is incompressible in $(M, S)$ if the inclusion map of $\tilde{F}$ into $\tilde{M}$ induces a monomorphism of $\pi_{1}(\tilde{F})$ into $\pi_{1}(\tilde{M})$.

Suppose that $(M, S)$ is the factor manifold corresponding to $\Gamma \backslash H^{3}$ for some discrete $\Gamma \subseteq \mathrm{PSL}_{2}(\mathbf{C})$. Let $F$ be a surface that intersects $S$ nicely. Let $\Phi$ be a component of the inverse image of $F$ in $H^{3}$. Then the stabilizer of $\Phi$ in $\Gamma$ is conjugate to the image of $\pi_{1}(\tilde{F})$ in $\pi_{1}(\tilde{M}) \cong \Gamma$.

PROPOSITION 1.8. Let $F$ be a nicely embedded two-sided surface in $(M, S)$. If $F$ is incompressible then $\pi_{1}(\tilde{M})$ is either an amalgamated sum over $\pi_{1}(\tilde{F})$ or an HNN group over $\pi_{1}(\tilde{F})$, depending on whether $F$ is separating or not.

PROOF. Proposition 1.8 is a direct application of the Seifert-Van Kampen Theorem.

2. Some splittings of the Bianchi groups. In this section we show that all of the Bianchi groups except $\Gamma_{3}$ split as amalgamated sums. Solitar $[\mathbf{S}]$ has shown that $\Gamma_{3}$ does not admit a splitting as an amalgamated sum. In Theorem 2.1 we summarize our findings for $\Gamma_{d}$ when $O_{d}$ is Euclidean. The splitting given for $\Gamma_{1}$ is common knowledge, our reference is $[\mathbf{F l}]$. The splittings for $\Gamma_{2}, \Gamma_{7}, \Gamma_{11}$ were indicated by incompressible annuli, although the actual presentations were derived from existing presentations. In Theorem 2.2 ve show that for $d \neq 1,3, \Gamma_{d}$ is an HNN group having amalgamated subgroup isomorphic to $\mathrm{PSL}_{2}(\mathbf{Z})$. This is achieved by showing that the factor manifold for $\mathrm{PSL}_{2}(\mathbf{Z})$ embeds as a nonseparating incompressible surface in $\Gamma_{d} \backslash H^{3}$. In Theorem 2.4 we show that when $O_{d}$ is non-Euclidean, $\Gamma \cong E_{d} *_{F_{d}} G_{d}$ where $E_{d}$ is the projective elementary matrices and $F_{d}$ is a particular subgroup corresponding to a nicely embedded separating incompressible sphere in $\Gamma_{d} \backslash H^{3}$ having four branch points, two of index 2 and two of index 3. When $O_{d}$ is Euclidean $\Gamma_{d} \cong E_{d}$, so that this surface cannot yield a nontrivial splitting. Although arrived at constructively our original hope was to use valuation theoretic methods as in [CS] to derive the existence of nontrivial splittings as amalgamated sums. It is interesting to note the similarity of the splitting $\Gamma_{d} \cong E_{d} *_{F_{d}} G_{d}$ and the splitting of Nagao's theorem, $\mathrm{GL}_{2}(K[f]) \cong \mathrm{GL}_{2}(k) *_{B(k)}$ $B(k[f])$.

In the following $D_{2}$ will denote the Klein four-group, $S_{3}$ the symmetric group on three letters, and $A_{4}$ the alternating group.

THEOREM 2.1. The Bianchi groups $\Gamma_{1}, \Gamma_{2}, \Gamma_{7}$, and $\Gamma_{11}$ are nontrivial free products with amalgamation. In particular

(i) $\Gamma_{1}=G_{1} *_{H} G_{2}$ where
(a) $G_{1}=S_{3} * Z_{3} A_{4}$,
(b) $G_{2}=S_{3} * Z_{2} D_{2}$ and
(c) $H=\mathrm{PSL}_{2}(\mathbf{Z})$; 
(ii) $\Gamma_{2}=G_{1} *_{H} G_{2}$ where

(a) $G_{1}$ is an $H N N$ group whose base is $D_{2}$ with two elements of order 2 amalgamated,

(b) $G_{2}$ is an $H N N$ group whose base is $A_{4}$ with two three-cycles amalgamated,

(c) $H=Z * Z_{2}$;

(iii) $\Gamma_{7}=G_{1} *_{H} G_{2}$ where

(a) $G_{1}=Z * Z_{2}$

(b) $G_{2}$ is an $H N N$ group with base $K=S_{3} * z_{3} S_{3}$ with two three-cycles, one from each $S_{3}$, amalgamated,

(c) $H=Z * Z_{2} * Z_{2}$;

(iv) $\Gamma_{11}=G_{1} *_{H} G_{2}$ where

(a) $G_{1}=Z * Z_{3}$,

(b) $G_{2}$ is an $H N N$ group with base $A_{4} * Z_{3} A_{4}$ and two three-cycles, one from each $A_{4}$, amalgamated,

(c) $H=Z * Z_{3} * Z_{3}$.

PrOOF. The decomposition of $\Gamma_{1}$ appears in [Fi1]. Because of the similarity of the computations we will describe how we arrived at the presentation for $\Gamma_{2}$. We will then give the cases $\Gamma_{7}$ and $\Gamma_{11}$ a light brushing over.

In [Fi2] the following presentation of $\Gamma_{2}$ is given, where $\mu=z+\omega, a=-1 / z$ and $t=z+1$ :

$$
\Gamma_{2}=\left\langle\mu, a, t ; a^{2}=(a t)^{3}=(\mu a \bar{\mu} a)^{2}=[t, \mu]=1\right\rangle .
$$

Letting $s=a t, v=\bar{\mu} s \mu$ and $m=\bar{\mu} a \mu$ and applying the appropriate Tietze transformations we get $\Gamma_{2}=\left\langle\mu, a, s, v, m ; a^{2}=s^{3}=m^{2}=(a m)^{2}=(s \bar{v})^{2}=\right.$ $1, \bar{\mu} a=m, a m=s \bar{v}, \bar{\mu} s=v\rangle$. From this presentation we see that $\Gamma_{2}$ is the amalgamated sum of $G_{1}=\left\langle a, m, u ; a^{2}=m^{2}=(a m)^{2}=1, \bar{\mu} a \mu=m\right\rangle$ and $G_{2}=\left\langle s, v, \mu ; s^{3}=v^{3}(s \bar{v})^{2}=1, \bar{u} s=v\right\rangle$ with identifications $\mu=\mu$ and $s \bar{v}=a m$. Clearly $G_{1}$ is an HNN extension of the Klein four-group with two elements of order two identified, and $G_{2}$ is an HNN extension of $A_{4}$ with two three-cycles identified. To complete the proof it must be shown that $\langle a m, \mu\rangle$ is isomorphic to $\langle s \bar{v}, \mu\rangle$ under the given homomorphism and that these two groups are isomorphic to $Z * Z_{2}$. Suppose that an HNN group is given with base group $G$ and free part $\mu$ and amalgamated subgroups $H, K$; that is $\bar{\mu} H \mu=K$. It is a consequence of of the normal form theorem for HNN groups that if $L$ is a subgroup of the base group that has trivial intersection with the amalgamated subgroups $H$ and $K$ then the group $\langle L, \mu\rangle$ is the free product of $L$ and $\langle\mu\rangle$. This applies in both the situations above, hence the proof is complete.

In [Fi2] the following presentation for $\Gamma_{7}$ is given:

$$
\Gamma_{7}=\left\langle a, v, s, m, w ; a^{2}=v^{3}=(a v)^{2}=1, a v=m s^{2}, \bar{w} a w=m, \bar{w} s w=v\right\rangle,
$$

where $a=-1 / z, m=(-\omega z+1-\omega) /(z+\omega), v=((1-\omega) z+1) /(z+\omega), s=$ $-1 /(z+1)$, and $w=-1 /(z+\omega)$. Letting $t=w^{2}$ and $x=a v$ and making the appropriate Tietze transformations we get $\Gamma_{7}=\left\langle a, v, w, t, x ; a^{2}=v^{3}=(a v)^{2} x^{2}=\right.$ $\left.1, w x w=a t \bar{v}, t=w^{2}, x=a v\right\rangle$. From this we see that $\Gamma_{7}$ is the amalgamated product of $G_{1}=\left\langle x w ; x^{2}=1\right\rangle$ and $G_{2}=\left\langle a, v, t ; a^{2}=v^{3}=(a v)^{2}(a t \bar{v} \bar{t})^{2}=1\right\rangle$ with 
identifications $x=a v, w^{2}=t$ and $w x w=a t \bar{v}$. The rest is similar to the above computations.

Interpretating $\{1, \omega\}$ to be the standard module basis for $\Gamma_{11}$ instead of $\Gamma_{7}$ we have that $\Gamma_{11}=\left\langle a, v, s, m ; a^{2}=s^{3}=(a v)^{3}=1, a v=s m, \bar{w} a w=m, \bar{w} s w=v\right\rangle$ where $a, v, s, m$, and $w$ have the formulas given above. The same substitutions as we made in the $\Gamma_{7}$ case yield the appropriate presentation.

THEOREM 2.2. If $d \neq 1,3$ then $\Gamma_{d}$ is an HNN group with amalgamated subgroup $\mathrm{PSL}_{2}(\mathbf{Z})$.

Proof. Let $H^{2}$ be im $z=0$ in $H^{3}$. Clearly the stabilizer of $H^{2}$ in $\Gamma_{d}$ contains $\mathrm{PSL}_{2}(\mathbf{Z})$. From [Sw, Lemma 8.2] we have that $B \cap H^{2}$ coincides with the Ford domain of $\mathrm{PSL}_{2}(\mathbf{Z})$ acting on $H_{2}$. Since a fundamental domain for the stabilizer of $H^{2}$ is the intersection of its Ford domain (in $\mathrm{H}^{2}$ ) and a fundamental domain for its subgroup of elements fixing infinity, we have that the stabilizer of $\mathrm{H}^{2}$ is generated by $\mathrm{PSL}_{2}(\mathbf{Z})$ and some transformations that fix infinity. Except for $\Gamma_{1}$ and $\Gamma_{3}$ this means that the additional transformations are of the form $z+\gamma$ where $\gamma \in O_{d}$. If such a map stabilizes $H^{2}, \gamma$ must be in $\mathbf{Z}$. Thus we have that for $d \neq 1,3$ the stabilizer of $H^{2}$ is $\mathrm{PSL}_{2}(\mathbf{Z})$. To show that the image of $H^{2}$ in $\Gamma_{d} \backslash H^{3}$ is embedded we must show that if $\sigma\left(H^{2}\right) \cap H^{2} \neq \varnothing$ for some $\sigma \in \Gamma_{d}$ then $\sigma\left(H^{2}\right)=H^{2}$. Suppose that $\sigma\left(H^{2}\right) \cap H^{2} \neq \varnothing$; we may precompose and postcompose $\sigma$ by elements of $\mathrm{PSL}_{2}(\mathbf{Z})$ to obtain $\tau$ so that $\tau$ carries a point in the Ford domain of $\mathrm{PSL}_{2}(\mathbf{Z})$, in $H^{2}$, to another point in the Ford domain of $\operatorname{PSL}_{2}(\mathbf{Z})$. Since the Ford domain for $\mathrm{PSL}_{2}(\mathbf{Z})$ lies inside the Ford domain for $\Gamma_{d}$ we have that $\tau$ fixes infinity. As above $\tau(z)=z+\gamma$. Since $\tau\left(H^{2}\right) \cap H^{2} \neq 0$ we have that $\gamma \in Z$. Thus $\tau\left(H^{2}\right) \cap H^{2}=H^{2}$ and consequently $\sigma\left(H^{2}\right)=H^{2}$. Hence the image of $H^{2}$ in $\Gamma_{d} \backslash H^{3}$ is an embedded two-sided surface $\Sigma$. From the structure of $H^{2} \cap B$ we infer that $\Sigma$ is a proper open disk intersecting the branch set nicely in two points, one of index 2 and one of index 3. Let $\tilde{\Sigma} \subset \overparen{\Gamma_{d} \backslash H^{3}}$ be the associated complexes as in $\S 1$. We get the presentation $\pi_{1}(\tilde{\Sigma})=\left\langle x_{1}, x_{2} ; x_{1}^{2}=x_{2}^{3}=1\right\rangle$. From the considerations above we see that we can choose basepoints so that $\pi_{1}(\tilde{\Sigma}) \rightarrow \pi_{1}\left(\widetilde{\Gamma_{d} \backslash H^{3}}\right) \rightarrow \Gamma_{d}$ induces a surjection $\pi_{1}(\tilde{\Sigma}) \rightarrow \mathrm{PSL}_{2}(\mathbf{Z})$. Since all nonelementary Fuchsian groups are Hopfian, this implies that the inclusion map $\pi_{1}(\tilde{\Sigma}) \rightarrow \pi_{1}\left(\widetilde{\Gamma_{d} \backslash H^{3}}\right)$ is a monomorphism. Therefore $\Sigma$ is incompressible. Let $\alpha$ be a straight line from $(0,2)$ to $(\omega, 2)$ in $H^{3}$. The arc $\alpha$ has been chosen so that it lies in the Ford domain of $\Gamma_{d}$. Its endpoints lie in $H^{2}$ and the translate of $H^{2}$ by $z+\omega$, so that $z+\omega$ maps one endpoint to the other, and its interior is disjoint from the translates of $H^{2}$. This allows us to conclude that the image of $\alpha$ in $\Gamma_{d} \backslash H^{3}$ is a simple closed curve intersecting $\Sigma$ in a single point of transverse intersection. Hence $\Sigma$ is nonseparating. The result now follows from Proposition 1.8.

Our next goal is to show that when $O_{d}$ is not Euclidean then $\Gamma_{d}$ splits as an amalgamated sum. To do this we need the following lemma.

LEMMA 2.3. Suppose $d \neq 1,2,3,7,11$, let

$$
\begin{gathered}
x_{1}= \pm\left(\begin{array}{cc}
0 & -1 \\
1 & 0
\end{array}\right), \quad x_{2}= \pm\left(\begin{array}{cc}
0 & -1 \\
1 & -1
\end{array}\right), \quad x_{3}= \pm\left(\begin{array}{cc}
\omega & -\omega^{2}-1 \\
1 & -1
\end{array}\right) \\
x_{4}= \pm\left(\begin{array}{cc}
-\omega & \omega^{2}-\omega+1 \\
-1 & -1+\omega
\end{array}\right) .
\end{gathered}
$$


The subgroup $F_{d}$ of $\mathrm{PSL}_{2}\left(O_{d}\right)$ generated by $x_{1}, x_{2}, x_{3}, x_{4}$ has the presentation

$$
\left\langle x_{1}, x_{2}, x_{3}, x_{4} ; x_{1}^{2}=x_{2}^{3}=x_{3}^{2}=x_{4}^{3}=x_{1} x_{2} x_{3} x_{4}=1\right\rangle .
$$

PROOF. Let $a= \pm\left(\begin{array}{cc}0 & -1 \\ 1 & 0\end{array}\right), t= \pm\left(\begin{array}{ll}1 & 1 \\ 0 & 1\end{array}\right)$, and $\mu=\left(\begin{array}{ll}1 & \omega \\ 0 & 1\end{array}\right)$. Then in terms of these the above four transformations are given by $x_{1}=a, x_{2}=a t, x_{3}=\mu a \mu^{-1}$, $x_{4}=\mu a t \mu^{-1}$. We note that $x_{1}, x_{2}$ generate the classical modular group $\mathrm{PSL}_{2}(\mathrm{Z})$.

From a result of Fine [Fi1], for any $d \neq 1,2,3,7,11$ the elements $a, t, \mu$ generate the projective elementary group $P E_{2}\left(O_{d}\right)=E_{d}$ and a complete presentation for $E_{d}$ is given by

$$
E_{d}=\left\langle a, t, \mu ; s^{2}=(a t)^{3}=[t, \mu]=1\right\rangle .
$$

Furthermore this presentation is independent of $d$.

In $E_{d}$, consider the normal closure of the modular group. We can denote this by $N(a, t)$. Then $E_{d} / N(a, t) \cong\langle\mu\rangle$. Therefore $\left\{\mu^{n}\right\}, n \in \mathbf{Z}$, constitutes a complete set of coset representatives for $N(a, t)$ in $E_{d}$. Using these and applying the Reidemeister-Schreier rewriting process we have that $N(a, t)$ is generated by $a_{i}=\mu^{i} a \mu^{-i}$ and $t_{i}=\mu^{i} t \mu^{-i}$ where $i$ ranges over $\mathbf{Z}$, with the relations $a_{i}^{2}=\left(a_{i} t_{i}\right)^{3}=1$ and $t_{i}=t_{i+1}$. Let $G_{i}=\left\langle a_{i}, t_{i} ; a_{i}^{2}=\left(a_{i} t_{i}\right)^{3}=1\right\rangle$. Then $N(a, t)$ is an infinite tree product with the $G_{i}$ 's as vertices and amalgamations, $t_{i}=t_{i+1}$.

In a tree product a presentation for a connected subtree is the obvious one, so for the subgroup generated by $G_{0}$ and $G_{1}$,

$$
\left\langle G_{0}, G_{1}\right\rangle=\left\langle a_{0}, a_{1}, t_{0}, t_{1} ; a_{0}^{2}=a_{1}^{2}=\left(a_{0} t_{0}\right)^{3}=\left(a_{1} t_{1}\right)^{3}=1, t_{0}=t_{1}\right\rangle .
$$

Noting that $a_{0}=a=x_{1}, a_{1}=\mu a \mu^{-1} x_{2}, a_{0} t_{0}=x_{3}$ and $a_{1} t_{1}=x_{4}$ we have that the subgroup of $\Gamma_{d}$ generated by $x_{1}, x_{2}, x_{3}$ and $x_{4}$ has presentation $\left\langle x_{1}, x_{2}, x_{3}, x_{4} ; x_{1}^{2}=\right.$ $\left.x_{3}^{3}=x_{2}^{2}=x_{4}^{3}=x_{1} x_{2} x_{3} x_{4}=1\right\rangle$.

THEOREM 2.4. When $d \neq 1,2,3,7,11, \Gamma_{d}$ admits a nontrivial splitting as $E_{d} *_{F_{d}} G_{d}$ where $E_{d}$ and $F_{d}$ are as above.

PROOF. Let $\Sigma$ denote the image of $H^{2}$ in $\Gamma_{d} \backslash H^{3}$. We can truncate the cusps of $\Gamma_{d} \backslash H^{3}$ to obtain a compact manifold $M_{d}$ having tori as boundary so that $\Sigma \cap M_{d}$ is a proper disk intersecting the branch set nicely in two points. Let $T_{\infty}$ denote the component of $\partial M_{d}$ having nonempty intersection with $\Sigma$. Let $N\left(\Sigma \cup T_{\infty}\right)$ be a small regular neighborhood and let $F$ be the frontier of $N\left(\Sigma \cup T_{\infty}\right)$. Notice that $F$ is a separating sphere in $\Gamma_{d} \backslash H^{3}$ that intersects the branch set nicely in four points, two of index 2 and two of index 3 . Let $\tilde{F}$ and $\overparen{\Gamma_{d} \backslash H^{3}}$ be the associated complexes. From the construction of $\tilde{F}$ we see that $\pi_{1}(\tilde{F}) \cong\left\langle x_{1}, x_{2}, x_{3}, x_{4} ; x_{1}^{2}=x_{2}^{3}=x_{3}^{2}=x_{4}^{3}=\right.$ $\left.1, x_{1} x_{2} x_{3} x_{4}=1\right\rangle$. The group corresponding to $N\left(\Sigma \cup T_{\infty}\right)$ is $E_{d}$ and the group corresponding to its frontier is $F_{d}$. This can be seen by considering the inverse image of $N\left(\Sigma \cup T_{\infty}\right)$ in $H^{3}$. From Lemma 2.4 and the fact that nonelementary Fuchsian groups are Hopfian we conclude that $\pi_{1}(\tilde{F}) \rightarrow \pi_{1}\left(\widetilde{\Gamma_{d} \backslash H^{3}}\right)$ is injective. By Proposition $1.8, \Gamma_{d} \cong E_{d} * F_{d} G_{d}$. Since $O_{d}$ is not Euclidean, $E_{d}$ is a proper subgroup of $\Gamma_{d}[\mathrm{Co}]$; hence $F_{d}$ is a proper subgroup of $G_{d}$ and the splitting is nontrivial. 


\section{BIBLIOGRAPHY}

[B1] L. Bianchi, Sui gruppi de sostituzioni lineari con coefficienti appartenenti a corpi quadratici imaginari, Math. Ann. 40 (1897), 332-412.

[B] M. Baker, Ramified primes and the homology of the Bianchi groups, Inst. Hautes Études Sci., preprint, 1982.

[Co] P. Cohn, On the structure of $\mathrm{GL}_{2}$ of a ring, Inst. Hautes Études Sci. Publ. Math. 30 (1966), 5-53.

[CS] M. Culler and P. Shalen, Bounded separating surfaces in knot manifolds, Invent. Math. 75 (1984), 537-545.

[Fi1] B. Fine, The structure of $\mathrm{PSL}_{2}(R)$, Ann. of Math. Studies, no. 79, Princeton Univ. Press, Princeton, N. J., 1974, pp. 145-170.

[F12] - The HNN and generalized free product structure of certain linear groups, Bull. Amer. Math. Soc. 81 (1975), 413-416.

[Fl] D. Flöge, Zur Struktur der $\mathrm{PSL}_{2}$ über einegen imaginär-quadratischen Zahlringen, Math. Z. 183 (1983), 255-279.

[GS] F. Grünewald and J. Schwermer, Arithmetic quotients of hyperbolic 3-space, cusp forms and link complements, Duke Math J. 48 (1981), 183-200.

[H] A. Hatcher, Bianchi orbifolds of small discriminant, an informal report, Preprint.

[R] R. Riley, Applications of a computer implementation of Poincaré's theorem on fundamental polyhedra, Math. Comp. 40 (1983), 607-632.

[Ro] J. Rohlfs, On the cuspidal cohomology of the Bianchi modular group, Math. Z. 188 (1985), 253-270.

[S] D. Solitar, private correspondence.

[Sw] R. Swan, Generators and relations for certain special linear groups, Adv. in Math. 6 (1871), 1-78.

[Th] W. Thurston, The geometry and topology of 3-manifolds, mimeographed notes.

[V] K. Voightmann, Rational homology of Bianchi groups, Math. Ann. (to appear).

[Z] R. Zimmert, Zur $\mathrm{SL}_{2}$ der ganzen Zahlen eines imaginär-quadratischen Zahlkörpers, Invent. Math. 19 (1973), 73-81.

[Z1] H. Zieschang, Finite groups of mapping classes of surfaces, Lecture Notes in Math., vol. 875, Springer-Verlag, Berlin and New York, 1981.

\section{Department of Mathematics, University of California, Santa Barbara, CALIFORNIA 93106}

Current address (Charles Frohman): Department of Mathematics, University of California at Los Angeles, Los Angeles, California 90024

Current address (Benjamin Fine): Department of Mathematics, Fairfield University, Fairfield, Connecticut 06430 